\title{
Catalyzing rapid discovery of gold-precipitating bacterial lineages with university students
}

\author{
Noah G. Riley ${ }^{1}{ }^{,}$,arlos C. Goller ${ }^{\text {Corresp., } 1,2}{ }^{,}$Zakiya H. Leggett ${ }^{3}$, Danica M. Lewis ${ }^{4}$, Karen Ciccone $^{4}$, Robert R. Dunn ${ }^{5,6}$ \\ 1 Department of Biological Sciences, North Carolina State University, Raleigh, NC, United States \\ 2 Biotechnology Program (BIT), North Carolina State University, Raleigh, NC, United States \\ 3 Department of Forestry and Environmental Resources (FER), North Carolina State University, Raleigh, NC, United States \\ 4 North Carolina State University Libraries, North Carolina State University, Raleigh, NC, United States \\ 5 Department of Applied Ecology, North Carolina State University, Raleigh, NC, United States \\ 6 University of Copenhagen, Natural History Museum of Denmark, Copenhagen, Denmark \\ ${ }^{7}$ German Centre for Integrative Biodiversity Research (iDiv), Halle-Jena-Leipzig, Germany \\ Corresponding Author: Carlos C. Goller \\ Email address: ccgoller@ncsu.edu
}

Intriguing and potentially commercially useful microorganisms are found in our surroundings, and new tools allow us to learn about their genetic potential and evolutionary history. Engaging students from different disciplines and courses in the search for microbes requires an exciting project with innovative but straightforward procedures and goals. Here we describe an interdisciplinary program to engage students from different courses in the sampling, identification, and analysis of the DNA sequences of a unique yet common microbe, Delftia spp. A campus-wide challenge was created to identify the prevalence of this genus, able to precipitate gold, involving introductory level environmental and life science courses, upper-level advanced laboratory modules taken by undergraduate students (juniors and seniors), graduate students, and staff from the campus. The number of participants involved allowed for extensive sampling while undergraduate researchers and students in lab-based courses participated in the sample processing and analyses, helping contextualize and solidify their learning of the molecular biology techniques. The results were shared at each step through publicly accessible websites and workshops. This model allows for the rapid discovery of Delftia presence and prevalence and is adaptable to different campuses and experimental questions. 


\section{Catalyzing rapid discovery of gold-precipitating bacterial 2 lineages with university students}

3

4

5

Noah G. Riley ${ }^{1}$, Carlos C. Goller ${ }^{1,2}$, Zakiya Leggett ${ }^{3}$, Danica Lewis ${ }^{4}$, Karen Ciccone ${ }^{4}$, Robert R. Dunn $^{5,6,7}$

${ }^{1}$ Department of Biological Sciences, North Carolina State University, Raleigh, NC, USA

2 Biotechnology Program (BIT), North Carolina State University, Raleigh, NC, USA

${ }^{3}$ Department of Forestry and Environmental Resources (FER), North Carolina State University, Raleigh, NC, USA

${ }^{4}$ North Carolina State University Libraries, North Carolina State University, Raleigh, NC, USA

${ }^{5}$ Department of Applied Ecology, North Carolina State University, Raleigh, NC, USA

${ }^{6}$ Natural History Museum of Denmark, University of Copenhagen, 2100 Copenhagen $\varnothing$, Denmark

${ }^{7}$ German Centre for Integrative Biodiversity Research (iDiv) Halle-Jena-Leipzig, Germany

Corresponding Author:

Carlos C. Goller ${ }^{1,2}$

6104 Jordan Hall, Raleigh, NC, 27695 USA

Email address: ccgoller@,ncsu.edu 
46

47

48

49

50

51

52

53

54

55

56

57

58

59

60

61

62

63

64

65

66

67

68

69

70

71

72

73

74

75

76

77

78

79

80

81

82

83

84

85

86

87

88

89

90

91

\section{Abstract}

Intriguing and potentially commercially useful microorganisms are found in our surroundings, and new tools allow us to learn about their genetic potential and evolutionary history. Engaging students from different disciplines and courses in the search for microbes requires an exciting project with innovative but straightforward procedures and goals. Here we describe an interdisciplinary program to engage students from different courses in the sampling, identification, and analysis of the DNA sequences of a unique yet common microbe, Delftia spp. A campus-wide challenge was created to identify the prevalence of this genus, able to precipitate gold, involving introductory level environmental and life science courses, upper-level advanced laboratory modules taken by undergraduate students (juniors and seniors), graduate students, and staff from the campus. The number of participants involved allowed for extensive sampling while undergraduate researchers and students in lab-based courses participated in the sample processing and analyses, helping contextualize and solidify their learning of the molecular biology techniques. The results were shared at each step through publicly accessible websites and workshops. This model allows for the rapid discovery of Delftia presence and prevalence and is adaptable to different campuses and experimental questions.

\section{Introduction}

The potential benefits from the study of the unique abilities of bacteria to everyday human life is ever more obvious. Bacteria are used industrially in food preparation, drug production, waste treatment, and many other roles. Advances in biotechnology techniques have facilitated the use of known bacterial species and their enzymes, proteins, and pathways (Berini, Casciello, Marcone, \& Marinelli, 2017). For example, it is now possible, and indeed not very difficult, to identify genes of interest in a bacterial species, clip those genes out of that species, and insert them into another work horse species of bacteria to allow the products of those genes to be produced industrially. Ironically, as our ability to harness the power of bacteria becomes ever more sophisticated, one of the key challenges is still finding the useful bacteria in the first place. In a world with as many as a trillion bacterial species (Locey \& Lennon, 2016)(Pike, Viciani, \& Kumar, 2018), how does one speed the discovery of bacterial species with a particular use or even simply strains of a particular bacterial taxon with sequences of interest?

One approach is to engage citizen scientists. In as much as the first step in the discovery of novel, useful microbes is often collections from nature, collections made by the public have the potential to speed up this key, and often rate-limiting, first step. What is more, in a rapidly interconnected digital era, the potential for truly global projects that rely on hundreds, thousands, or even hundreds of thousands of individuals is ever greater (Cooper, 2016). Citizen scientists contribute data to many publicly-accessible projects, from birdwatchers helping conservation efforts with the e-Bird project (https://ebird.org/home; Sullivan et al., 2014), game enthusiasts folding proteins for the FoldIt project (https://fold.it/portal/; Cooper et al., 2010), or homeowners exploring the microbial diversity in their houses (http://robdunnlab.com/projects/wild-life-ofour-homes/; Dunn, 2013). Additionally, projects like the Science Education Alliance - Phage Hunters Advancing Genomics and Evolutionary Science (SEA-PHAGES) and Tiny Earth engage students in large research projects as part of course-based undergraduate research experiences (CUREs) (Hanauer, et al., 2017)(Handelsman, J., 2018). Citizen scientists, we argue, can also help discover bacteria with novel, useful traits. 
92

93

94

95

96

97

98

99

100

101

102

103

104

105

106

107

108

109

110

111

112

113

114

115

116

117

118

119

120

121

122

123

124

125

126

127

128

129

130

131

132

133

134

135

136

137

Delftia is a genus first discovered in the city Delft (Den Dooren de Jong, 1927; Wen, Fega, Hayward, Chakraborty, and Sly 1999), where bacteria themselves were discovered by Leeuwenhoek (Gest, 2004). Delffia have genes capable of precipitating gold by excreting a metabolite called delftibactin (Johnston et al., 2013). Gold in solution as gold chloride is toxic to bacteria, so Delftia has evolved this novel mechanism for precipitating aqueous gold out of solution to non-toxic solid gold nanoparticles. This mechanism has obvious potential uses in gold recycling in used electronics, gold mining, and urban waste ("Gold Recycling," 2013; Reith, Lengke, Falconer, Craw, \& Southam, 2007; Subhabrata, Natarajan, \& Ting, 2017), but to date, the existing genetic diversity of Delftia in strain collections is modest. There are only six known species of Delftia. Full genome assemblies exist for four of these species within the National Center for Biotechnology Information (NCBI) database (Wen, Fegan, Hayward, Chakraborty, \& Sly, 1999). Discovery of novel Delftia species and their relatives has the potential to better elucidate variations in Delftia genetic sequences, especially within the gold precipitation gene cluster and other industrially and human health related sequences. The more information about these gold precipitation genes, for example, the greater potential for using Delftia or its genetic potential to recycle our electronics and make mining more sustainable.

Here we leverage a citizen science approach to detect new Delftia species on a university campus. We simultaneously test whether students are able to aid the speed of discovery of novel lineages and consider the biology of the lineages we have discovered. The Wolfpack Citizen Science Challenge for spring 2018 (go.ncsu.edu/wpc18) was a collaborative project to document the presence and genetic diversity of Delftia spp. across the North Carolina State University campus and create a scalable and interdisciplinary model to continue learning about this and other organisms. In addition to involving students in two introductory courses in the initial data collection, we also involved students in two upper-level courses in the downstream study of the microbes detected during the Challenge.

\section{Materials \& Methods}

\section{Recruitment of Participants and Sample Collection}

Participants were primarily recruited from two courses, ES 100: Introduction to Environmental Sciences (176 students) and LSC 170: First Year Seminar in the Life Sciences: Meet Your Microbes (20 students). However, anyone interested was able to obtain a sampling kit and participate. A post-event survey indicated that $96 \%$ of the participants were required to participate as part of a course, and that $48 \%$ were currently enrolled as STEM majors.

Three events were held to create excitement and share results from the challenge. In January, the Challenge was launched with a public event attended by 19 people, in which Goller and Riley shared information about Delftia acidovorans found in sinks, drains, and soil and encouraged members of the campus to think critically about the microbial communities around us. In March, the sequencing data were shared with the campus community at an event at which participants used the NCBI Basic Local Alignment Search Tool (BLAST) to find regions of similarity between the discovered sequences and those deposited in the NCBI database. This BLAST workshop was attended by 55 people. In April, results of the project were shared at a closing event open to the campus and general public, attended by 30 people.

Participants registered as teams of up to five members and were provided kits with instructions and materials to collect samples: three swabs and two $50 \mathrm{~mL}$ conical tubes for soil samples along with gloves, plastic spoons for scooping soil, alcohol swabs to sanitize the soil 
138 collection spoons, and labels for samples. Approximately forty kits were distributed, and over 139 one hundred and fifty swab and soil samples were received between January 30 and February 14.

140 Samples were delivered in person to either the Biotechnology Program (BIT) teaching

141 laboratories or the NC State University Libraries front desk. Samples were stored in $-20^{\circ} \mathrm{C}$

142 freezer until ready for metagenomic DNA extraction. Along with physical samples, metadata

143 including location descriptors and latitude-longitude data were submitted online through a

144 customized SciStarter citizen science website (https://scistarter.com/delftia). Students'

145 identifying information was removed from samples and a numerical identity was assigned.

146

147

\section{Safety}

Participants were provided with detailed instructions on how to sample environments around the campus and use the sampling kit. Participants were instructed to use the swab to sample a safe location and immediately place the swab in the transport container. Students collected soil samples with the provided tube and spoon while wearing disposable gloves. For processing of samples, students in molecular biology courses were trained in lab safety procedures and given a document detailing the potential hazards and safety procedures used in the teaching laboratory. For all extractions and qPCR reactions, students wore provided disposable lab coats, safety glasses, and gloves, and disinfected all surfaces before and after use.

\section{Isolation and Purification of Metagenomic DNA}

Metagenomic DNA was extracted from samples using the Invitrogen PureLink Microbiome DNA Purification Kit according to the corresponding protocol for swab and soil samples, respectively. Soil was transferred from collection tubes to bead tubes with alcoholsterilized metal scoops. Swab tips were cut off into bead tubes with alcohol-sterilized metal scissors. Samples were lysed and homogenized by heat, bead beating, and lysis buffer. After purification, samples were eluted in $50 \mu \mathrm{l}$ of elution buffer. DNA concentration was determined spectrophotometrically using a ThermoFisher NanoDrop 2000c instrument and normalized to 5 $\mathrm{ng} / \mu \mathrm{l}$. Samples were matched with descriptive location data in an online spreadsheet using information submitted on the SciStarter website (https://scistarter.com/delftia). Isolations were performed by Riley in batches of 12-24 samples.

\section{Detection of Delftia-specific Sequences by Quantitative Real-time PCR}

An Eppendorf epMotion 5075 TC liquid handler was used to set up quantitative real-time PCR (qPCR) reactions with New England BioLabs Luna Universal Probe qPCR reagents, primers, and double-quenched probes (IDT DNA). qPCR reactions were run on a Bio-Rad CFX Connect instrument, and data were exported as spreadsheets with cycle threshold $(\mathrm{Ct})$ values for each reaction. Samples were screened for the quantity of Delftia present using double-quenched, Delftia-specific primers and probe for a portion of the unique gold biomineralization metabolite production system (hereafter "gold gene"; Johnston et al. 2013; GenBank CP000884.1, region 5233319-5234363; see Supplemental Data S1 for primer and probe sequences, Seq1, Seq2, Seq3). Presence and abundance of Delftia were then confirmed with a second set of primers and probe for a putative Delftia-specific toxin-antitoxin sequence unique to Delftia spp. (hereafter "CP sequence"; GenBank CP000884.1, region 759992-760309; see Supplemental Data S1 for primer and probe sequences, Seq4, Seq5, Seq6). Reactions were set up in duplicate along with an 8-point, ten-fold dilution standard curve with "Gold Gene" standard beginning at $40 \mathrm{pg} / \mu \mathrm{l}$ and 
$183 \mathrm{CP}$ gene standard at $30 \mathrm{pg} / \mu 1$. Dilution calculation tables and $\mathrm{qPCR}$ conditions are described in 184 Supplemental Data S2.

185

186

187

188

189

190

191

192

193

194

195

196

197

198

199

200

201

202

203

204

205

206

207

208

209

210

211

212

213

214

215

216

217

218

219

220

221

222

223

224

225

226

227

228

\section{Abundance Estimation of Delftia spp. in Samples}

Undergraduate juniors and seniors and first- and second-year graduate students enrolled in an upper-level High-throughput Discovery 8-week lab module programmed an epMotion 5075 TC liquid handler with the qPCR script, prepared metagenomic samples for qPCR, and calculated Delftia copy numbers using the qPCR Ct data (see Supplemental Table S1). Students were provided a spreadsheet template with detailed explanations and information on the use of a standard curve for calculation of absolute copy numbers of target sequences. Data were shared with students, and groups of three to four were tasked with determining copy numbers for one 96-well PCR plate containing: twenty-three genomic DNA samples tested in duplicate along with an 8-point standard curve and negative "buffer only" controls. Multiple groups analyzed the same samples to confirm the results, and copy number trends were further supported by analyzing qPCR data for the same samples with a primer set for the single-copy Delftia-specific "CP" sequence described above. Data were then analyzed as a class and shared with Danica Lewis (NCSU Libraries) for visualization and dissemination of the results to participants and the public (go.ncsu.edu/exploredelftia). Samples with the highest Delftia copy number using both primer sets were selected for further analysis of the unique "gold" sequence.

Sequencing of "Gold Gene" in Samples Positive for Delftia spp.

For twenty samples with high Delftia counts, a portion of the gold gene sequence was amplified using primers Seq7 and Seq8 identified in Supplemental Data S1 and the Q5(R) High-Fidelity 2X Master Mix (New England Biolabs) according to the protocol outlined in Supplemental Data S3. The amplified portion of the gold gene was selected because it is highly specific to Delftia and, based on current sequence database information, varies slightly between known species and strains, allowing for identification from metagenomic samples. The target Delftia sequence is 1045 base pairs in length (Supplemental Data S4). Of the twenty tested samples, seventeen produced sufficient PCR product for sequencing and were sent to the NC State University Genomic Sciences Laboratory (GSL) for Sanger DNA sequencing using primers Seq7 and Seq8. Amplicons were sequenced from both directions, and sequences were trimmed based on stringent quality settings to match existing sequences in the NCBI database. The sequencing data were shared with the campus community at an event at which participants used the NCBI Basic Local Alignment Search Tool (BLAST) to find regions of local similarity between the discovered sequences and those deposited in the NCBI database. This allowed participants to identify which Delftia species and strains best matched the samples that were sequenced (see Supplemental Data S5-S9).

\section{Data Dissemination}

The Google Maps Fusion Tables extension was used to create a heatmap of Delftia presence and abundance across campus, and Tableau Public software was used to create an interactive map (http://go.ncsu.edu/ExploreDelftia). Participants were invited to explore the data and evaluate which samples had the highest amount of Delftia. Students in the courses involved in sampling and analysis were shown the results and asked to discuss future research questions.

Peer] reviewing PDF | (2019:11:43148:1:1:NEW 9 Mar 2020) 
229

230

231

232

233

234

235

236

237

238

239

240

241

242

243

244

245

246

247

248

249

250

251

252

253

254

255

256

257

258

259

260

261

262

263

264

265

266

267

268

269

270

271

272

273

274

\section{Results}

Proportion of Samples Containing Delftia spp. Sequences

Over 150 samples were received from participants. Of these, 135 were labeled correctly and matched with the online SciStarter database containing sampling location descriptions and latitude-longitude coordinates. Through qPCR analysis using primers and probe Seq1, Seq2, and Seq3, 125 samples $(92.6 \%)$ had detectable quantities of the target Delftia "gold gene" DNA sequence. Quantities of Delftia within samples were confirmed using the CP qPCR primers and probe Seq4, Seq5, and Seq6. The twenty samples with highest Delftia counts were primarily swabs from sinks and drains (Table 1). In contrast, the samples with the least Delftia DNA tended to be those from soil samples and outdoor locations. However, it is worth reiterating that nearly all of the samples contained some Delftia, a relatively understudied genus of bacteria.

We next compared the Delftia gold gene sequences in the samples to those of sequenced strains. Collectively, the sequences from our samples were most similar to those of Delftia tsuruhatensis strain CM13, Delftia acidovorans strains ANG1 and SPH-1, or Delftia acidovorans strain RAY209 (see Table 2). Differentiation between D. acidovorans strains ANG1 and SPH-1 was not possible as each matched query had the same identity, query coverage, and E value results for both strains. However, for strains of D. tsuruhatensis CM13 and D. acidovorans RAY209, the sequences matched with highest probability to each, respectively. None of our samples were close matches for the other sequenced Delftia species of D. deserti, D. lacustris, D. litopenaei, D. rhizosphaerae, or other strains of D. acidovorans and D. tsuruhatensis. Fourteen out of the seventeen sequences had less than $97 \%$ sequence identity with the Delftia strains they most closely matched.

\section{Discussion}

Here, we sought to simultaneously test whether we could engage students campus-wide in a citizen science style microbial research project and, in doing so, understand the distribution and diversity of strains of one particular bacterial genus, Delftia. In short, we were indeed able to engage students from diverse majors across campus. In doing so, we discovered that some sampling sites had many more Delftia counts than did others, that Delftia was relatively ubiquitous, and that some of the strains we identified had gold genes that appeared relatively divergent from those known from the literature. Although we were unable to accurately determine the diversity of Delftia strains present, this unanswered question presents a new challenge and opportunity for our citizen science and Delftia research efforts.

Collectively, the qPCR, Sanger DNA sequencing, and BLAST comparison results showed that strains of Delftia are diverse, abundant and frequent (found at many sites) in environments in and around the college campus. Based on available genomic sequences deposited in the NCBI database and partial sequencing of the highly conserved gold gene, the strains students discovered beset matched the reference strains D. tsuruhatensis CM13 and D. acidovorans ANG1 and SPH-1. However, fourteen of seventeen samples contained strains that were a $97 \%$ or lower match to strains in the NCBI database. Our suspicion is that these strains represent uncharacterized genetic diversity among strains in Delftia's gold gene. However, because we sequenced from complex environmental samples we can't preclude the possibility that some of this variation is due to cases in which the forward and reverse sequences obtained were from different Delftia species or strains in the sample. 
275

276

277

278

279

280

281

282

283

284

285

286

287

288

289

290

291

292

293

294

295

296

297

298

299

300

301

302

303

304

305

306

307

308

309

310

311

312

313

314

315

316

317

318

319

320

The sequenced Delftia gold gene from many of the participant samples matched well to known Delftia species, but some samples matched two different existing strains equally well. For example, samples from 7-1 and 24-1 were equally similar to the strains D. acidovorans ANG1 and SPH-1 (Table 2). Clearly further work can be done to sequence additional portions or the entire genomes of these samples to identify what known strain is present or discover a new lineage of Delftia. More extensive community analyses of the samples using both targeted (16S rRNA gene) and whole genome shotgun sequencing would aid in the identification of which microbes associate with the presence of Delftia and the identity of the gold sequences in the environment, respectively. Additionally, high-throughput sequencing approaches such as Hi-C from Phase Genomics ("Hi-C Proximity-Guided Assembly," 2018)(Sieber et al., 2018) or Nanopore single-molecule long-read sequencing can be employed to attempt to sequence and assemble the entire Delftia genome in metagenomic samples positive for Delftia by qPCR. Ultimately, selective media capable of isolating and identifying Delftia would allow us to increase our collection of Delftia strains for basic functional studies and genome sequencing.

Our sequencing results best matched the species $D$. acidovorans and D. tsuruhatensis, both of which have been found in environments similar to those we studied. D. acidovorans was originally discovered in soil and has been found in drains, waterspouts, and showerheads in the built environment (Wen, Fegan, Hayward, Chakraborty, \& Sly, 1999). D. tsuruhatensis was first discovered in a wastewater treatment plant and has been found in similar locations along with $D$. acidovorans (Hou et al., 2015). The Delftia species we did not encounter in our study are species that have so far been associated with more restricted habitats. Delftia deserti has been found to inhabit desert environments (Li et al., 2015), D. lacustris in lake water (Jørgensen, Brant, Nybroe, \& Hansen, 2012), D. litopenaei in pond water (Chen, Lin, Sheu, \& Sheu, 2012), and D. rhizosphaerae in the rhizosphere of Cistus ladanifer, a plant native to the Mediterranean region (Carro et al., 2017). The apparent ubiquity of the genus Delftia hides the reality that individual species appear to show considerable habitat restriction. In the future, it would be interesting to understand which traits and genes of individual Delftia species confer the ability to survive in particular habitats.

It is unclear the extent to which the life history of Delftia in the above habitats is the same as that of Delftia in the built environment of a college campus. Nor is it well understood whether the presence of Delftia in water systems is problematic or potentially beneficial. Like many bacterial taxa, Delftia species are recorded as opportunistic pathogens that can infect hospitalized or immunocompromised patients (Patel et al., 2019)(Bilgin, Sarmis, Tigen, Soyletir, \& Mulazimoglu, 2015). However, there is no indication that human bodies are a common habitat for this genus. Instead, in buildings such as those we sampled it appears to be much more common in water systems - in drains, showerheads, and downspouts. In as much as the ecological conditions of water systems differ greatly, it is possible that a comparative study of water systems, such as those that are or are not chlorinate,) might reveal more about the build environment natural history of this organism (cite our NTM study).

Our approach kindled campus-wide student interest in microbial diversity and molecular biology techniques through the excitement of discovering this unique microbe in places that students frequent on campus. Groups of students from various academic disciplines and courses produced and analyzed samples that contributed to a large public dataset. The findings helped teach the student community about Delftia, and also reinforced the importance of the collaborative nature of scientific discovery. The success of this project, in terms of the documentation of Delftia's distribution helps to validate our general approach. In addition, our

Peer] reviewing PDF | (2019:11:43148:1:1:NEW 9 Mar 2020) 
321 approach has the potential to encourage future students to participate. We aim to continue the

322

323

324

325

326

327

328

329

330

331

332

333

334

335

336

337

338

339

340

341

342

343

344

345

346

347

348

349

350

351

352

353

354

355

356

357

358

359

360

361

362

363

364

365

366 challenge of accurately identifying new Delftia lineages and engage others by expanding the sampling opportunity to a multi-section first-year English class that is required for all undergraduate students on our campus. Using a similar approach and incorporating the expertise of faculty in the English department, we will engage students in writing tasks related to the project. Additionally, an upper-level metagenomics course will tie into this endeavor by processing, sequencing, and analyzing the microbial communities in samples with high numbers of Delftia sequences. With relatively minor changes to the course schedules and curricula, one hundred more students per semester can participate, learn, and contribute to the project. We are creating resources that are accessible for other faculty and campuses to implement this project and share findings. For this, students participating in the project are writing the Delftia book (go.ncsu.edu/delftiabook), and we have created a group for instructor resources on the QUBES web portal (https://qubeshub.org/community/groups/delftia/projects). Liquid handlers can be cost-prohibitive, but less expensive models such as the Opentrons OT-2 are available, and we are developing scripts for this instrument. Student groups in lab-based courses can always set up qPCRs manually to participate in this project.

\section{Conclusions}

As the future plans for integrating this project into courses indicate, enthusiasm for the project was high among our colleagues and grew as the project proceeded. However, if we are to continue the project it is key that it continues to yield new scientific insights. Fortunately, this seems very likely to be the case. For example, although Delftia abundance was very patchy on campus, we have yet to explain what factors account for such patchiness. Additional samples will help us to have sufficient coverage across sample types to allow spatial models of Delftia diversity and abundance. In addition, our results suggest that new variants of the Delftia gold gene and even new Delftia strains remain to be discovered. Conversely, there is a lack of genomic diversity represented in the NCBI database. By leveraging the enthusiasm of university students and staff, interconnecting courses and researchers, and using our model pipeline, new lineages of Delftia can be rapidly identified and studied (e.g., groups of students cloning novel gold gene cluster into a host such as Escherichia coli or yeast for functional characterization). This will yield a better understanding of the ecological and environmental significance of these organisms and simultaneously help to connect students and faculty across campus in a common scientific project. Finally, it is of note that Delftia species, while little known, are of potentially great applied importance. In addition, they contain genes that allow many strains to precipitate gold. Given the many waste streams in which gold is present but hard to concentrate, this ability has the potential to be very useful moving forward.

\section{Acknowledgements}

We would like to acknowledge the contributions of all the students involved in this project and the support offered by the NC State Biotechnology Program and Genomic Sciences Laboratory.

Peer] reviewing PDF | (2019:11:43148:1:1:NEW 9 Mar 2020) 
367

368

369

370

371

372

373

374

375

376

377

378

379

380

381

382

383

384

385

386

387

388

389

390

391

392

393

394

395

396

397

398

399

400

401

402

403

404

405

406

407

\section{References}

Berini, F., Casciello, C., Marcone, G.L., \& Marinelli, F. (2017). Metagenomics: Novel enzymes from non-culturable microbes. FEMS Microbiology Letters, 364(21), fnx211.

Bilgin, H., Sarmis, A., Tigen, E., Soyletir, G., \& Mulazimoglu, L. (2015). Delftia acidovorans: A rare pathogen in immunocompetent and immunocompromised patients. Canadian Journal of Infectious Diseases and Medical Microbiology, 26(5), 277-279.

Carro, L., Mulas, R., Pastor-Bueis, R., Blanco, D., Terrón, A., González-Andrés, F., Peiz, A., Velázquez, E. (2017). Delftia rhizosphaerae sp. nov. isolated from the rhizosphere of Cistus ladanifer. International Journal of Systematic and Evolutionary Microbiology, 67(6), 1957-1960.

Chen, W.M., Lin, Y.S., Sheu, D.S., \& Sheu, S.Y. (2012). Delftia litopenaei sp. Nov., a poly- $\beta-$ hydroxybutyrate-accumulating bacterium isolated from a freshwater shrimp culture pond. International Journal of Systematic and Evolutionary Microbiology, 62(Pt 10), 23152321.

Conniff, R. (2012). A bitter pill. Conservation Magazine.

Cooper, C. (2016). Citizen science: How ordinary people are changing the face of discovery. New York, NY: The Overlook Press.

Cooper, S., Khatib, F., Treuille, A., Barbero, J., Lee, J., Beenen, M., ... Foldit players. (2010). Predicting protein structures with a multiplayer online game. Nature, 466, 756-760.

Den Dooren de Jong, L. E. (1927). Ueber Protaminophage Bakterien. Zentralbl. Bakteriol. Parasitenkd. Infektionskr. Hyg. Abt. 2 71, 193-232.

Dunn, R.R., Fierer, N., Henley, J.B., Leff, J.W., \& Menninger, H.L. (2013). Home life: Factors structuring the bacterial diversity found within and between homes. PloS ONE, 8(5): e64133.

Gest, H. (2004). The discovery of microorganisms by Robert Hooke and Antoni van Leeuwenhoek, Fellows of the Royal Society. Notes and Records of the Royal Society of London, 58(2), 187-201.

Gold recycling: Using delftibactin to recycle gold from electronic waste. (2013). iGem Team Heidelberg.

Hanauer, D.I., Graham, M.J., SEA-PHAGES, Betancur, L., Bobrownicki, A., Cresawn, S.G., Garlena, R.A., Jacobs-Sera, D., Kaufmann, N., Pope, W.H., Russell, D.A., Jacobs Jr., W.R., Sivanathan, V., Asai, D.J., \& Hatfull, G.F. An inclusive Research Education Community (iREC): Impact of the SEA-PHAGES program on research outcomes and student learning. Proceedings of the National Academy of Sciences, 114(51), 1353113536.

Handelsman, J., Hernandez, S., Tsang, T., Bascom-Slack, C., \& Broderick, N. (2018). Tiny Earth - A Reseach Guide to Studentsourcing Antibiotic Discovery. Ann Arbor, MI: XanEdu Publishing Inc.

Hou, Q., Wang, C., Guo, H., Xia, Z., Ye, J., Liu, K., Yang, Y., Hou, X., Liu, H., Wang, J., Du, B., \& Ding, Y. (2015). Draft genome sequence of Delftia tsuruhatensis MTQ3, a strain of 
408

409

410

411

412

413

414

415

416

417

418

419

420

421

422

423

424

425

426

427

428

429

430

431

432

433

434

435

436

437

438

439

440

441

plant growth-promoting rhizobacterium with antimicrobial activity. Genome Announcements, 3(4), e00822-15. doi: 10.1128/genomeA.00822-15

Johnston, C.W., Wyatt, M.A., Li, X., Ibrahim, A., Shuster, J., Southam, G., \& Magarvey, N.A. (2013). Gold biomineralization by a metallophore from a gold-associated microbe. Nature Chemical Biology, 9(4), 241-243.

Jørgensen, M.O., Brandt, K.K., Nybroe, O., \& Hansen, M. (2015). International Journal of Systematic and Evolutionary Microbiology, 59(Pt 9), 2195-2199.

Li, C.T., Yan, Z.F., Chu, X., Hussain, F., Xian, W.D., Yunuz, Z., Hozzein, W.N., Abaydulla, G., \& Li, W.J. (2015). Delftia deserti sp. nov., isolated from a desert soil sample. Antonie Van Leeuwenhoek, 107(6), 1445-1450.

Locey, K.J., \& Lennon, J.T. (2016). Scaling laws predict global microbial diversity. Proceedings of the National Academy of Sciences, 113(21), 5970-5975.

Patel, D., Igbal, M., Mubarik, A., Vassa, N., Godil, R., Saad, M., Muddassir, S. (2019). Delftia acidovorans: A rare cause of septic pulmonary embolism from catheter-related infection: Case report and literature review. Respiratory Medicine Case Reports, 12, 27.

Pike, L.J., Viciani, E., \& Kumar, N. (2018). Microbial diversity knows no borders. Nature Review Microbiology, 16, 66.

Reith, F., Lengke, M., Falconer, D., Craw, D., \& Southam, G. (2007). The geomicrobiology of gold. The ISME Journal, 1(7), 567-584.

Segata, N., Izard, J., Waldron, L., Gevers, D., Miropolsky, L., Garrett, W. S., \& Huttenhower, C. (2011). Metagenomic biomarker discovery and explanation. Genome Biology, 12(6), R60.

Sieber, C.M.K., Probst, A.J., Sharrar, A., Thomas, B.C., Hess, M., Tringe, S.G., \& Banfield, J.F. (2018). Recovery of genomes from metagenomes via a dereplication, aggregation and scoring strategy. Nature Microbiology, 3, 836-843.

Subhabrata, D., Natarajan, G., \& Ting, Y. (2017). Bio-extraction of precious metals from urban solid waste. AIP Conference Proceedings, 1805, 020004.

Sullivan, B.L., Aycrigg, J.L., Barry, J.H., Bonney, R.E., Bruns, N., Cooper, C.B., ... Kelling, S. (2014). The eBird enterprise: An integrated approach to development and application of citizen science. Biological Conservation, 169, 31-40.

Wen, A., Fegan, M., Hayward, C., Chakraborty, S., \& Sly, L.I. Phylogenetic relationships among members of the Comamonadaceae, and description of Delftia acidovorans (den Dooren de Jong 1926 and Tamaoka et al. 1987) gen. Nov., comb. Nov. (1999). International Journal of Systematic Bacteriology, 49(2), 567-576. 


\section{Table $\mathbf{1}$ (on next page)}

Top twenty samples with Delftia DNA identified via qPCR targeting of unique "gold" gene. 
1 Table 1: Top twenty samples with Delftia DNA identified via qPCR targeting of unique "gold" 2 gene.

\begin{tabular}{|c|c|c|c|c|c|c|}
\hline $\begin{array}{c}\text { DNA } \\
\text { Sample } \\
\text { Number }\end{array}$ & $\begin{array}{c}\text { Delftia } \\
\text { Gold } \\
\text { Gene } \\
\text { Count }\end{array}$ & Latitude & Longitude & $\begin{array}{c}\text { Sample } \\
\text { Type }\end{array}$ & Location & Description \\
\hline $15-1$ & 113,191 & 35.78593062 & 78.66805315 & Swab & Poe Hall & Water fountain \\
\hline $7-1$ & 17,294 & 35.78472956 & 78.67292404 & Swab & $\begin{array}{l}\text { Owen } \\
\text { Residence Hall }\end{array}$ & None provided \\
\hline $24-1$ & 14,167 & 35.78822065 & 78.67522672 & Swab & $\begin{array}{l}\text { University } \\
\text { Towers }\end{array}$ & $\begin{array}{l}\text { parking deck } \\
\text { drains }\end{array}$ \\
\hline $1-3$ & 12,041 & 35.78654 & -78.671737 & Swab & Williams Hall & bathroom sink \\
\hline $17-3$ & 9,493 & 35.78068018 & 78.67308866 & Swab & $\begin{array}{l}\text { Wood } \\
\text { Residence Hall }\end{array}$ & Sink drain \\
\hline $23-2$ & 8,780 & 35.78468 & -78.666723 & Swab & SAS building & $\begin{array}{l}\text { The girls bathroom } \\
\text { sink on the first } \\
\text { floor of SAS } \\
\text { building, middle } \\
\text { sink }\end{array}$ \\
\hline $33-2$ & 5,789 & 35.78744498 & 78.67013454 & Swab & $\begin{array}{l}\text { DH Hill } \\
\text { Library }\end{array}$ & $\begin{array}{l}\text { 3rd floor Women's } \\
\text { bathroom sink }\end{array}$ \\
\hline $12-2$ & 5,095 & 35.785385 & -78.673091 & Swab & $\begin{array}{l}\text { Metcalf } \\
\text { bathroom }\end{array}$ & bathroom sink \\
\hline $26-1$ & 5,095 & 35.74477072 & 78.68757963 & Swab & $\begin{array}{l}\text { Campus } \\
\text { Crossing }\end{array}$ & $\begin{array}{l}\text { Apartment } \\
\text { complex }\end{array}$ \\
\hline $9-1$ & 4,612 & 35.78795303 & 78.67699295 & Swab & $\begin{array}{l}\text { Valentine } \\
\text { Commons }\end{array}$ & Kitchen sink \\
\hline $44-4$ & 4,356 & 35.78670028 & 78.67463044 & Soil & $\begin{array}{l}\text { fence on Dan } \\
\text { Allen Dr. }\end{array}$ & $\begin{array}{l}\text { chilly (56 F), drier } \\
\text { soil, live } \\
\text { organisms present }\end{array}$ \\
\hline $25-2$ & 2,961 & 35.7861221 & 78.66352558 & Swab & $\begin{array}{l}\text { NCSU Bell } \\
\text { Tower, main } \\
\text { campus }\end{array}$ & $\begin{array}{l}\text { Wild Card sample- } \\
\text { seat located on } \\
\text { NCSU bell tower }\end{array}$ \\
\hline $45-4$ & 2,317 & 35.78753054 & 78.67083426 & Soil & Atrium & $\begin{array}{l}\text { Trash bins next to } \\
\text { the vending } \\
\text { machines }\end{array}$ \\
\hline $24-2$ & 1,971 & 35.78822065 & $\begin{array}{r}- \\
78.67522672 \\
\end{array}$ & Swab & $\begin{array}{l}\text { University } \\
\text { Towers }\end{array}$ & drain \\
\hline $15-2$ & 1,873 & 35.785982 & -78.677831 & Swab & Lee Hall & Suite 807 Sink \\
\hline
\end{tabular}




\begin{tabular}{|r|r|r|r|l|l|l|}
\hline $18-1$ & 1,733 & 35.78481659 & 78.67285967 & Swab & $\begin{array}{l}\text { Owen residence } \\
\text { hall }\end{array}$ & $\begin{array}{l}\text { inside in dorm } \\
\text { room }\end{array}$ \\
\hline $25-1$ & 1,535 & 35.77153404 & & & $\begin{array}{l}\text { Engineering } \\
\text { Building I, } \\
\text { Centennial } \\
\text { Campus }\end{array}$ & $\begin{array}{l}\text { Drain in the } \\
\text { middle of the floor } \\
\text { of the bathroom }\end{array}$ \\
\hline $29-2$ & 1,452 & 35.78751407 & 78.66981704 & Swab & DH Hill & Floor 1 \\
\hline $7-2$ & 1,381 & 35.78412031 & 78.67101431 & Swab & $\begin{array}{l}\text { Talley Student } \\
\text { Union }\end{array}$ & $\begin{array}{l}\text { Bathroom Sink } \\
\text { Drain }\end{array}$ \\
\hline $30-2$ & 1,113 & 35.78824567 & 78.67403984 & Swab & Nelson Hall & Water fountain \\
\hline
\end{tabular}

3 
Table 2 (on next page)

NCBI BLAST results for sequenced environmental "gold gene". 
1 Table 2: NCBI BLAST results for sequenced environmental "gold gene".

\begin{tabular}{|c|c|c|c|c|}
\hline Sample & Species and Strain & Identity & Query Coverage & E Value \\
\hline $1-3$ & \multirow{11}{*}{$\begin{array}{l}\text { Delftia tsuruhatensis } \\
\text { strain CM13 }\end{array}$} & $98 \%$ & $100 \%$ & 0.0 \\
\hline $9-1$ & & $96 \%$ & $100 \%$ & 0.0 \\
\hline $12-2$ & & $95 \%$ & $99 \%$ & 0.0 \\
\hline $15-1$ & & $97 \%$ & $100 \%$ & 0.0 \\
\hline $15-2$ & & $95 \%$ & $100 \%$ & 0.0 \\
\hline $17-3$ & & $95 \%$ & $100 \%$ & 0.0 \\
\hline $25-1$ & & $95 \%$ & $100 \%$ & 0.0 \\
\hline $25-2$ & & $96 \%$ & $100 \%$ & 0.0 \\
\hline $26-1$ & & $96 \%$ & $100 \%$ & 0.0 \\
\hline $30-2$ & & $95 \%$ & $99 \%$ & 0.0 \\
\hline $33-2$ & & $97 \%$ & $100 \%$ & 0.0 \\
\hline $7-1$ & \multirow{4}{*}{$\begin{array}{l}\text { Delftia acidovorans } \\
\text { strain ANG1 or strain SPH-1 }\end{array}$} & $91 \%$ & $100 \%$ & 0.0 \\
\hline $18-1$ & & $94 \%$ & $99 \%$ & 0.0 \\
\hline $23-2$ & & $95 \%$ & $100 \%$ & 0.0 \\
\hline $24-1$ & & $95 \%$ & $100 \%$ & 0.0 \\
\hline $7-2$ & \multirow{2}{*}{$\begin{array}{l}\text { Delftia acidovorans } \\
\text { strain RAY209 }\end{array}$} & $82 \%$ & $99 \%$ & $3 e-154$ \\
\hline $30-3$ & & $96 \%$ & $99 \%$ & 0.0 \\
\hline
\end{tabular}

